\title{
A presentation of a recent case series on VACTERL associations
}

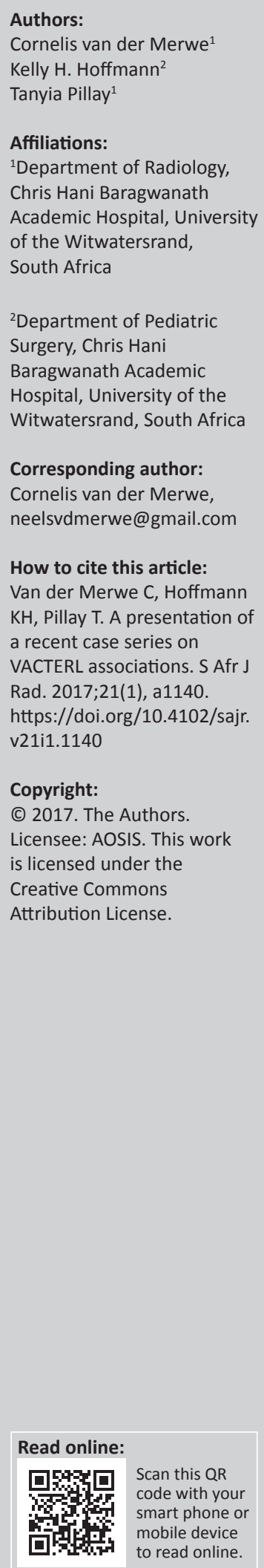

Note: A selection of conference abstracts: RSSA/SASPI Paediatric Imaging Congress, 03-06 November 2016, Spier Estate, Stellenbosch, Note: A selection of conference abstracts: RSSA/SASPI Paediatric Imaging Congress, 03-06 November 2016, Spier Estate, Stellenbosch, South Africa. Faculty collaborators. Professor Kassa Darge (Body Imaging, University of Pennsylvania, Philadelphia, USA), Professor USA), Pree (Thoracic lmaging, Havard University, USA), Professor Beverley Newman (Cardiac Imaging, Stanford University, California, USA), Professor Kimberly Applegate (Image Gently and Body Imaging, Emory University, Atlanta, USA) and Professor Savvas Andronikou (Thoracic Imaging, University of Bristol, UK) supported by South African Paediatric Radiologists, co-ordinated by Dr Jaishree Naidoo, President of the African Society of Paediatric Imaging and Head of Division of Paediatric Radiology, Charlotte Maxeke Johannesburg Academic Hospital.

Background: Vertebral defects, anal atresia, cardiac defects, tracheoesophageal fistula, renal anomalies, and limb abnormalities (VACTERL) association is described as the non-random cooccurrence of congenital malformations. The diagnosis of VACTERL association requires 3 component features of the following: vertebral, anorectal, cardiac, tracheoesophageal, renal or limb abnormalities, in the absence for evidence of another condition. The incidence of VACTERL association is reported as between 1 in 10000 to 1 in 40 000. The aetiology of VACTERL is still unclear, although the genetic spectrum is becoming somewhat better understood.

Object: This poster is an educational presentation on a case series of VACTERL association patients and the role radiology has in the identification of the associations.

Method: Images presented represents a case series of VACTERL association patients who presented to the Paediatric Surgery Department at the Chris Hani Baragwanath Academic Hospital in April and May 2016.

Conclusion: VACTERL association is reported to be an uncommon disorder. This case series highlights various imaging features of VACTERL association and proves the importance of screening for associated abnormalities in any patient presenting with one of the VACTERL associations. 